\title{
Orientational wetting and capillary nematization of hard-rod fluids
}

\author{
R. van RoiJ $\left(^{*}\right)$, M. Dijkstra( $\left(^{* *}\right)$ and R. Evans \\ H. H. Wills Physics Laboratory, University of Bristol - Bristol BS8 1TL, UK
}

(received 10 September 1999; accepted in final form 26 November 1999)

PACS. 64.60.Cn - Order-disorder transformations; statistical mechanics of model systems.

PACS. 64.70.Md - Transitions in liquid crystals.

PACS. 68.45.Gd - Wetting.

\begin{abstract}
We investigate the phase behavior of colloidal hard-rod fluids near a single wall and confined in a slit pore. Zwanzig's hard-rod model is analyzed by minimization of a free energy functional, and a system of freely rotating spherocylinders is studied by Monte Carlo simulations. Both theory and simulation show i) a wall-induced surface transition from uniaxial to biaxial symmetry, ii) complete orientational wetting of the wall-isotropic fluid interface by a nematic film, and iii) capillary nematization, with a capillary critical point, induced by confinement in the slit pore.
\end{abstract}

Fluids of nonspherical hard-core particles have attracted much attention over the years. The field was pioneered by Onsager in the 1940s, when he showed that a fluid of hard rodlike particles exhibits an isotropic-nematic (IN) phase transition upon increasing the density sufficiently [1]. A major step forward took place in the 1980s, when computer simulations, theories, and experiments, showed smectic, columnar, and other liquid crystalline phases in dense systems of hard rods or discs [2]. These developments showed that purely entropic (packing) effects arising from short-ranged steric repulsions are sufficient to drive bulk liquid crystalline behavior; long-ranged anisotropic dispersion forces are not essential for the occurrence of the various bulk phases. By contrast the nature of the orientational order in nonspherical hardcore fluids near a surface or in a confining pore is poorly understood. One can enquire, for instance, whether there are transitions analogous to (pre)wetting and capillary condensation that occur in simple fluids [3], or to prefreezing and exotic crystalline packing of hard spheres near walls [4], or whether there are transitions of a completely different nature. Given the present ability to produce (non)spherical colloidal particles by well-controlled synthesis [5], such issues are not only of theoretical interest but also within experimental reach. Moreover, knowledge of the behavior of nonspherical particles near surfaces is crucial for a better understanding of effective interactions between colloidal particles in a nematic solvent $[6,7]$. In this letter we report studies of hard-rod fluids near a single hard wall and confined by two parallel hard walls, i.e. a slit pore. Our theory and simulations do indeed reveal remarkably

$\left(^{*}\right)$ Present address: Institute for Theoretical Physics, University of Utrecht, The Netherlands.

$\left({ }^{* *}\right)$ Present address: Debye Institute, University of Utrecht, The Netherlands. 
rich phase behavior. This simple system displays a surface-induced orientational ordering transition, complete orientational wetting, and a first-order capillary nematization transition terminating in a capillary critical point. We first discuss the theory, and then the simulations.

An important technical complication in the theoretical description of inhomogeneous hardrod fluids in the isotropic or nematic phase is caused by the simultaneous presence of inhomogeneity and anisotropy in the one-particle distribution. In the present case of hard rods in contact with parallel hard walls, the loss of translational invariance may also break the uniaxial symmetry, and the most general one-particle distribution $\rho$ depends nontrivially on the center-of-mass coordinate $z$ perpendicular to the wall, and on the polar and azimuthal angle $\theta$ and $\varphi$, respectively, of the rod orientation with respect to the wall normal $\hat{z}$. In fact, even if one restricts attention to the uniaxially symmetric $\varphi$-independent regime (which as we will see is the low-density regime), the numerical task is far from trivial [6]. The difficulty involved in dealing with three coordinates instead of only one as in bulk (viz. $\theta$ ) is circumvented in the present theoretical analysis by employing Zwanzig's rod-model [8], where i) the rods have the shape of a $L \times D \times D$ rectangular block with $L \gg D$, and ii) the rod-orientations are restricted to the three orthogonal unit vectors $\hat{x}_{\alpha}$ with $\alpha=1,2,3$ representing $\hat{x}, \hat{y}$, and $\hat{z}$, respectively. The bulk phase behavior of this model was analyzed in 1963 by Zwanzig, who found a first-order IN transition similar to Onsager's result for freely rotating cylindrical rods [8]. Despite its simplicity this model captures the essential physics and key symmetries of the bulk IN transition. Here we show, by making comparison with simulations, that the same holds for surface and confinement phenomena in hard-rod fluids. We are concerned with the equilibrium one-particle distributions $\rho_{\alpha}(z)$ and the phase behavior of this system in given external potentials $V_{\alpha}(z)$ at a fixed chemical potential $\mu$, the total volume of the system being $V$, the spatial coordinate $z$, and the rod-orientation $\alpha=1,2,3$. To this end, we consider the grand-potential functional $\Omega[\rho]$ which is minimised by the equilibrium one-particle distribution, the minimal value being the equilibrium grand potential [9]. Within Onsager's second virial approximation, it is straightforward to show that the functional can be written, neglecting $\mathcal{O}(D / L)$ terms, as

$$
\begin{aligned}
\frac{\Omega[c] L^{2} D}{A k_{\mathrm{B}} T} & =\sum_{\alpha=1}^{3} \int c_{\alpha}(z)\left(\ln c_{\alpha}(z)-1+\beta\left(V_{\alpha}(z)-\mu\right)\right) \mathrm{d} z+ \\
& +2 \int\left(c_{1}(z) c_{2}(z)+\left(c_{1}(z)+c_{2}(z)\right) \bar{c}_{3}(z)\right) \mathrm{d} z
\end{aligned}
$$

with dimensionless density $c_{\alpha}(z)=L^{2} D \rho_{\alpha}(z)$, total (extensive) wall area $A$, inverse temperature $\beta=1 / k_{\mathrm{B}} T$, and $\bar{c}_{\alpha}(z)=(1 / L) \int_{z-L / 2}^{z+L / 2} \mathrm{~d} z^{\prime} c_{\alpha}\left(z^{\prime}\right)$. The Euler-Lagrange equations resulting from the minimum condition $\delta \Omega[c] / \delta c_{\alpha}(z)=0$ can be cast in the form

$$
\begin{aligned}
& \ln c_{1}(z)=\beta \mu-\beta V_{1}(z)-2 c_{2}(z)-2 \bar{c}_{3}(z) \\
& \ln c_{2}(z)=\beta \mu-\beta V_{2}(z)-2 c_{1}(z)-2 \bar{c}_{3}(z), \\
& \ln c_{3}(z)=\beta \mu-\beta V_{3}(z)-2 \bar{c}_{1}(z)-2 \bar{c}_{2}(z),
\end{aligned}
$$

which allows for straightforward iterative numerical solutions on a one-dimensional z-grid; results presented here are based on grids of 100 or 200 equidistant points per $L$, with some checks using 500 points per $L$. The profiles obtained from eqs. (2) are conveniently expressed in terms of the profiles of the total density $c=\sum_{\alpha=1}^{3} c_{\alpha}$, nematic order parameter $s=$ $\left[c_{3}-\left(c_{1}+c_{2}\right) / 2\right] / c$, and biaxial order parameter $\Delta=\left(c_{1}-c_{2}\right) / c$. 
In the absence of any external potential, i.e. for $V_{\alpha}(z)=0$, it is easily shown that the homogeneous bulk distribution $c(z)=c_{\mathrm{b}}, s(z)=s_{\mathrm{b}}$, and $\Delta(z)=0$ satisfies eqs. (2) with $\beta \mu\left(c_{\mathrm{b}}, s_{\mathrm{b}}\right)=\ln \left[c_{\mathrm{b}}\left(1+2 s_{\mathrm{b}}\right) / 3\right]+4 c_{\mathrm{b}}\left(1-s_{\mathrm{b}}\right) / 3$, provided $s_{\mathrm{b}}$ satisfies $2 c_{\mathrm{b}} s_{\mathrm{b}}=\ln \left[\left(1+2 s_{\mathrm{b}}\right) /\left(1-s_{\mathrm{b}}\right)\right]$. The resulting minimum value of the grand potential can then be written as $\Omega=-p V$, with the pressure $p$ given by $\beta p\left(c_{\mathrm{b}}, s_{\mathrm{b}}\right) L^{2} D=c_{\mathrm{b}}+2 c_{\mathrm{b}}^{2}\left(1-s_{\mathrm{b}}^{2}\right) / 3$. Bulk coexistence of an isotropic phase of density $c_{\mathrm{I}}$ and order parameter $s_{\mathrm{I}}=0$ and a nematic phase of density $c_{\mathrm{N}}$ and order parameter $s_{\mathrm{N}} \neq 0$ occurs when $\mu\left(c_{\mathrm{I}}, 0\right)=\mu\left(c_{\mathrm{N}}, s_{\mathrm{N}}\right)=\mu_{\mathrm{IN}}$ and $p\left(c_{\mathrm{I}}, 0\right)=p\left(c_{\mathrm{N}}, s_{\mathrm{N}}\right)=p_{\text {IN }}$; a straightforward numerical calculation yields, in accordance with Zwanzig's original results, the values $c_{\mathrm{I}}=1.258, c_{\mathrm{N}}=1.915, s_{\mathrm{N}}=0.915, \beta p_{\mathrm{IN}} L^{2} D=2.313$, and $\beta \mu_{\mathrm{IN}}=0.8087$. These results, known to at least 10 digits, are then used as input for analysis of the free planar IN interface, i.e. we consider eqs. (2) with $\mu=\mu_{\mathrm{IN}}$ and $V_{\alpha}(z)=0$, subject to the boundary conditions of coexisting isotropic and nematic bulk distributions at $z \rightarrow \pm \infty$, respectively. The resulting equilibrium profiles and the surface tension $\gamma_{\mathrm{IN}}=\left(\Omega+p_{\mathrm{IN}} V\right) / A$ depend on the relative orientation of the bulk nematic director $\hat{n}$ and the interface normal $\hat{z}$. In the biaxially symmetric geometry characterised by $\hat{n} \cdot \hat{z}=0$, we find $\beta \gamma_{\mathrm{IN}} L D=(2.7960 \pm 0.0004) \times 10^{-2}$, while the uniaxially symmetric geometry with $\hat{n} \cdot \hat{z}=1$ yields $\beta \gamma_{\text {IN }}^{\prime} L D=(5.0660 \pm 0.0005) \times$ $10^{-2}$. The prime denotes that the latter geometry is thermodynamically unfavorable compared to the former, in agreement with earlier results for the IN interface of freely rotating hard rods, where a tilt angle of $90^{\circ}$ between $\hat{n}$ and $\hat{z}$ corresponds to the lowest possible surface tension [10].

We now consider the fluid of Zwanzig rods, at chemical potential $\mu$, in contact with a single hard wall described by the external potential

$$
\beta V_{\alpha}^{(1)}(z)=\left\{\begin{array}{ccc}
\infty, & \text { for } \quad z<0 & (\alpha=1,2) \\
& z<L / 2 & (\alpha=3) \\
0, & \text { otherwise }
\end{array}\right.
$$

The immediate consequence of such a wall is that rods oriented perpendicular to the wall, i.e. rods characterised by $\alpha=3$, cannot approach closer than a center-of-mass distance $L / 2$. In other words, $c_{3}(z)=0$ and $s(z)=-1 / 2$ for $0 \leq z \leq L / 2$, as follows from eq. (2). The nature of the wall-induced orientational order is of interest now, since the distribution may be either uniaxially symmetric about $\hat{z}\left(\right.$ i.e. $c_{1}(z)=c_{2}(z)$ ), or biaxially symmetric (i.e. $\left.c_{1}(z) \neq c_{2}(z)\right)$. By considering appropriate linear combinations of eqs. (2), it follows that the biaxial order parameter profile $\Delta(z)$ satisfies

$$
\ln \frac{1-s(z)+\frac{3}{2} \Delta(z)}{1-s(z)-\frac{3}{2} \Delta(z)}=2 c(z) \Delta(z) .
$$

For any $s(z)$ and $c(z)$ there is a trivial uniaxial solution $\Delta(z)=0$ to eq. (4). Nontrivial biaxial solutions $\Delta(z) \neq 0$ exist at sufficiently high local density $c(z) \geq 3 /[2(1-s(z))]$, as follows from a low- $\Delta$ expansion of the logarithm in (4). Given that $s(z)=-1 / 2$ close to the wall, we conclude that wall-induced biaxiality $\Delta(z) \neq 0$ is possible for $c(z) \geq 1$, and thus sets in if $c_{1}(z)=c_{2}(z)=1 / 2$. The chemical potential $\mu_{\mathrm{UB}}$ at this uniaxial-biaxial (UB) bifurcation follows from eqs. (2), which for $z=0$ and $c_{1}(0)=c_{2}(0)=1 / 2$ yield $\beta \mu_{\mathrm{UB}}=1-\ln 2=0.307<\beta \mu_{\mathrm{IN}}$; the corresponding isotropic bulk fluid density is $c_{\mathrm{UB}}=1.031$. The relative "undersaturation" at which this wall-induced orientational surface ordering takes place can therefore be characterised by the ratio $c_{\mathrm{UB}} / c_{\mathrm{I}}=0.819$, which is not very different from the corresponding ratio 0.847 for freely rotating infinitely long spherocylinders at a hard wall as obtained by Poniewierski [11] from a bifurcation analysis of the Onsager freeenergy functional. Note, however, there is some fundamental difference between the in-plane 


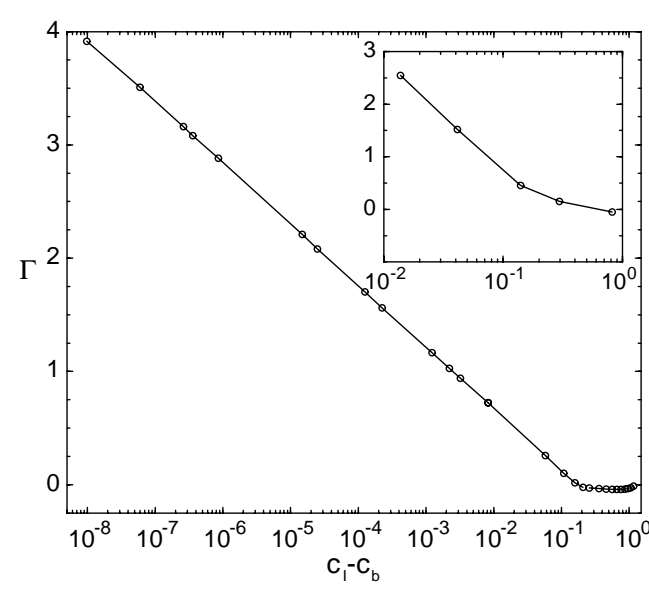

Fig. 1

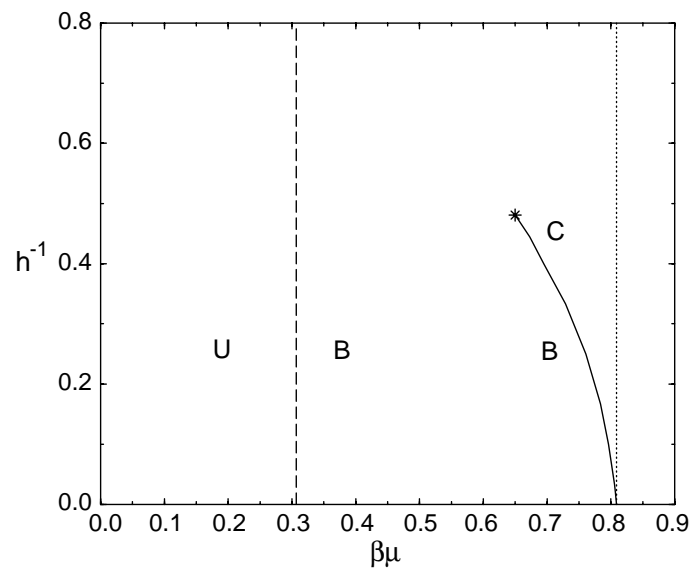

Fig. 2

Fig. 1 - The adsorption $\Gamma$ vs. bulk density $c_{\mathrm{b}}$. Main figure: second virial theory for Zwanzig model, showing a logarithmic divergence as $c_{\mathrm{b}} \rightarrow c_{\mathrm{I}}^{-}$, the isotropic coexistence density. Inset: simulation result for spherocylinders with $L / D=15$.

Fig. 2 - Phase diagram of confined Zwanzig hard-rod fluid as a function of chemical potential $\mu$ and wall separation $h$, showing uniaxially and biaxially symmetric phases $\mathrm{U}$ and $\mathrm{B}$, respectively, and a capillary nematic phase $\mathrm{C}$. The UB transition (dashed line) at $\beta \mu=0.307$ is continuous, whereas the capillary nematization or BC transition (solid curve) is first order and terminates in a capillary critical point $(*)$ at $h=h_{c}=2.08 \pm 0.01$. The vertical dotted line at $\beta \mu=0.8087$ marks the location of the bulk IN transition.

correlations for models with restricted orientations, such as the Zwanzig model, and those for models which are unrestricted. In the former, there should be true long-range order at the wall in the B phase, whereas in the latter the presence of a soft mode (presumably) destroys long-range, in-plane order. The repercussions for the nature of the UB transition warrant further study. Although there are some profound (symmetry) differences between the present continuous UB transition and the first-order prewetting transition (from a thin to a thick film) found in simple fluids near attractive walls, there is a similarity in the sense that the wall induces a surface transition (here the onset of orientational ordering, in simple fluids a condensation from a thin to a thick film) at a lower chemical potential than the corresponding bulk transition.

We now focus on the numerically determined biaxially symmetric equilibrium profiles that result from eqs. (2) with $\mu_{\mathrm{UB}}<\mu<\mu_{\mathrm{IN}}$. These profiles represent a nematic film with director $\hat{x}$ (or equivalently $\hat{y}$ ) close to the wall, and they decay into an isotropic distribution of density $c_{\mathrm{UB}}<c_{\mathrm{b}}<c_{\mathrm{I}}$ far from the wall. The thickness of the film, or equivalently the total adsorption $\Gamma=(1 / L) \int_{0}^{\infty} \mathrm{d} z\left[c(z)-c_{\mathrm{b}}\right]$, diverges logarithmically when $c_{\mathrm{b}} \rightarrow c_{\mathrm{I}}^{-}$, as is shown in fig. 1 . This divergence is a signature of complete wetting of the wall-isotropic fluid interface by the nematic phase (with a director perpendicular to the wall normal). This complete orientational wetting was not discussed in the work of Poniewierski [11] on hard spherocylinders, but is consistent with the findings of ref. [12] in the context of flexible polymers. The complete wetting scenario is confirmed by a numerical calculation, along the lines of that of $\gamma_{\mathrm{IN}}$ discussed above, of the surface tensions $\gamma_{\mathrm{WI}}$ and $\gamma_{\mathrm{WN}}$ associated with the wall-isotropic fluid (WI) and wallnematic fluid (WN) interface, respectively, at $\mu=\mu_{\mathrm{IN}}$. We find $\cos \vartheta=\left(\gamma_{\mathrm{WI}}-\gamma_{\mathrm{WN}}\right) / \gamma_{\mathrm{IN}}=$ $0.9999 \pm 0.0004$, consistent with complete wetting, contact angle $\vartheta=0$. 


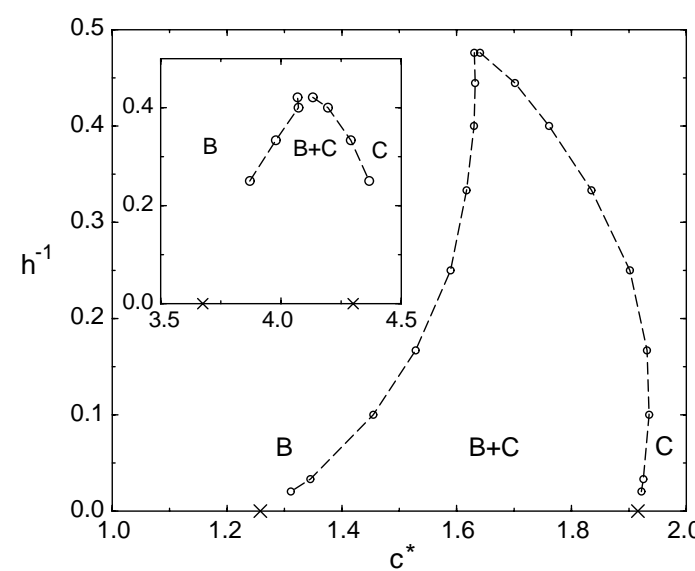

Fig. 3

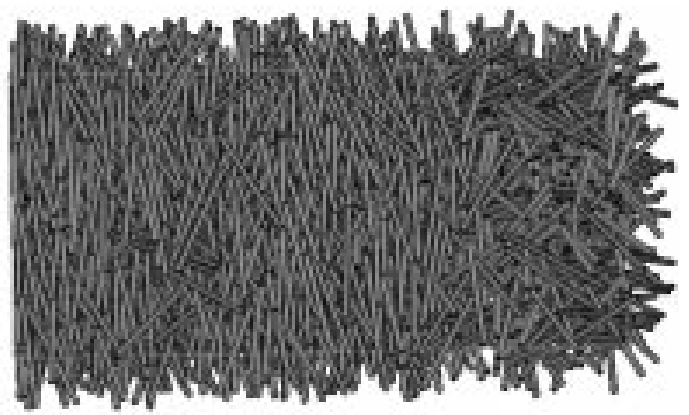

Fig. 4

Fig. 3 - Phase boundaries of biaxial (B) and capillary nematic (C) phases in $c^{*}-h^{-1}$ representation, clearly showing the critical point at $h=h_{c}=2.08 \pm 0.01$ and the approach to bulk IN coexistence $(\times)$ as $h^{-1} \rightarrow 0$. The inset shows the corresponding simulation results.

Fig. 4 - Equilibrium snapshot of about 1600 hard spherocylinders $(L / D=15)$ forming a nematic film between a hard wall (left) and an isotropic fluid (right).

Suppose now the spatial confinement is due to two parallel hard walls at $z= \pm H / 2$, described by the external potential $V_{\alpha}^{(2)}(z)=V_{\alpha}^{(1)}(H / 2-|z|)$, with the one-wall potential $V_{\alpha}^{(1)}(z)$ defined in eq. (3). We solve the Euler-Lagrange equations (2), with $V_{\alpha}(z)=V_{\alpha}^{(2)}(z)$, in the interval $-H / 2 \leq z \leq H / 2$ for given wall separation $H$ and chemical potential $\mu$. Insertion of the resulting equilibrium profiles into the functional yields the surface free energy $\gamma=(\Omega+p(\mu) V) / A$, with $V=A H$ and $p(\mu)$ the bulk pressure at chemical potential $\mu$. As in the one-wall system we find only profiles of uniaxial symmetry (with $\gamma=\gamma_{\mathrm{U}}$ ) when $\mu<\mu_{\mathrm{UB}}$, whereas additional biaxially symmetric profiles (with $\gamma=\gamma_{\mathrm{B}}$ ) exist for $\mu>\mu_{\mathrm{UB}}$. We find that $\gamma_{\mathrm{B}} \leq \gamma_{\mathrm{U}}$ for $\mu \geq \mu_{\mathrm{UB}}$, with the equality holding only, for any dimensionless wall separation $h=H / L$, if $\mu=\mu_{\mathrm{UB}}$. This $h$-independence of the continuous UB transition translates into the vertical dashed UB phase boundary at $\beta \mu=0.307$ in the $\mu-h^{-1}$ representation of the phase diagram shown fig. 2. In contrast, a strongly $h$-dependent transition is found for higher $\mu\left(<\mu_{\mathrm{IN}}\right)$ and sufficiently large $h$. Under these conditions a capillary nematic profile exists as a solution of eqs. (2), with $\gamma=\gamma_{\mathrm{C}}$. Unlike the uniaxial and biaxial profiles discussed above this capillary nematic profile does not decay towards an essentially isotropic distribution in the middle of the slit, but instead towards a relatively dense nematic distribution with a nematic director perpendicular to $\hat{z}$. Coexistence of a biaxial and a condensed nematic (C) phase takes place when $\gamma_{\mathrm{B}}=\gamma_{\mathrm{C}}$; this defines the capillary nematization curve in fig. 2. Strikingly, this BC coexistence ends in a capillary critical point $(*)$ at $h=h_{\mathrm{c}}=2.08 \pm 0.01$. Such a coexistence line is the analogue of the capillary condensation line found, for a given temperature, in simple fluids [3]. A capillary critical point is also found in a phenomenological Landau-de Gennes theory of thermotropic liquid crystals [13]. To the best of our knowlegde this behavior has not been predicted or observed in hard-core fluids. In order to facilitate comparison with the simulations to be discussed below, we show the BC coexistence in the $c^{*}-h^{-1}$ representation in fig. 3, where $c^{*}=(1 / H) \int_{-H / 2}^{H / 2} \mathrm{~d} z c(z)$ is the average density in the slit. Here the low- $c^{*}$ 
branch represents the B-phase and the high- $c^{*}$ branch the capillary nematic phase. In the limit $h^{-1} \rightarrow 0$ the $\mathrm{BC}$ coexistence curve smoothly approaches the bulk coexistence densities $c_{\mathrm{I}}$ and $c_{\mathrm{N}}$ indicated by $\times$. The peculiar shape of the coexistence curve reflects the fact that the difference in $c^{*}$ between the two phases was chosen as the order parameter. Other measures of the adsorption, for instance $\Gamma$ as a function of $\mu$ at fixed $h$, lead to more conventional shapes, with mean-field order parameter exponent $\beta=1 / 2$. We anticipate, as in the case of simple fluids [3], that this capillary criticality should lie in the 2D Ising universality class since the order parameter is a scalar and correlations can only diverge in the $x$ - $y$ plane.

In order to test the validity of the theoretical predictions we performed Monte Carlo simulations of a fluid of freely rotating hard spherocylinders of length-to-diameter ratio $L / D=15$. Again $c_{\mathrm{b}}$ denotes the dimensionless bulk density, which is now defined as $c_{\mathrm{b}}=(L+D)^{2} D \rho$ for number density $\rho$; the local density $c(z)$ is defined accordingly. We first determined the bulk IN coexistence by standard Gibbs ensemble simulations [14] of 2300 particles, which yield coexistence densities $c_{\mathrm{I}}=3.675 \pm 0.003$ and $c_{\mathrm{N}}=4.300 \pm 0.003$. Second we considered isotropic bulk fluids in contact with a single hard wall, where the isotropic distribution and bulk density $c_{\mathrm{b}}<c_{\mathrm{I}}$ far from the wall is imposed by umbrella sampling [14]. We measured the profile $c(z)$ and the $z$-dependent eigenvalues and eigenvectors of the standard $3 \times 3$ nematic order parameter tensor. For $c_{\mathrm{b}}<c_{\mathrm{UB}}=2.80 \pm 0.05$ we find that this matrix is of uniaxial symmetry for all $z$, with symmetry axis $\hat{z}$, whereas biaxiality sets in when $c_{\mathrm{b}}>c_{\mathrm{UB}}$. This suggests the occurrence of a symmetry breaking UB transition at $c_{\mathrm{UB}} / c_{\mathrm{I}}=0.76$, in qualitative agreement with the theoretical predictions for this ratio. But we emphasize again that the detailed nature of the transition remains to be ascertained. Further simulations for state points $c_{\mathrm{b}} \rightarrow c_{\mathrm{I}}^{-}$lend very strong support to the theoretical prediction of complete orientational wetting. This can be appreciated in the snapshot of fig. 4 , which shows an equilibrium nematic film of a thickness of about $4 L$ between the wall and the isotropic "bulk" fluid (here with thickness of merely $2 L$ or so) at $c_{\mathrm{b}} / c_{\mathrm{I}}=0.9887$. More quantitative support for complete orientational wetting is provided by the measured logarithmic increase of the adsorption $\Gamma$, shown in the inset of fig. 1 [15].

We tested the predictions of capillary nematization and its critical point by performing Gibbs ensemble simulations of the spherocylinders confined by two parallel hard walls at separations $h=H /(L+D)=4,3,2.5,2.375$, and 2.25. The resulting phase diagram, shown in the inset of fig. 3 in terms $h^{-1}$ and the average density $c^{*}$ in the slit, shows BC coexistence if $h \geq 2.375$ whereas no coexistence could be found for $h \leq 2.25$. The decrease of the density jump as $h$ is reduced, the overall shape of the coexistence curve, and the absence of coexistence for small $h$, are all features in agreement with the results of the Zwanzig model. They provide compelling evidence for capillary nematization with an accompanying capillary critical point in this hard-rod system.

This work was supported by grants ERBFMBICT971869 and EPSRC GR/L89013.

\section{REFERENCES}

[1] Onsager L., Ann. N.Y. Acad. Sci., 51 (1949) 627.

[2] Allen M. P., Evans G. T., Frenkel D. and Mulder B., Adv. Chem. Phys., 86 (1993) 1 and references therein.

[3] Evans R. and Marini Bettolo Marconi U., J. Chem. Phys., 86 (1987) 7138; Evans R., J. Phys. Condens. Matter, 2 (1990) 8989 and references therein. 
[4] Courtemanche D. J. and van Swol F., Phys. Rev. Lett., 69 (1992) 2078; Schmidt M. and Löwen H., Phys. Rev. Lett., 76 (1996) 4552; Phys. Rev. E, 55 (1997) 7228.

[5] Lekkerkerker H. N. W., Buining P. A., Buitenhuis J., Vroege G. J. and Stroobants A., Observation, Prediction, and Simulation of Phase Transitions in Complex Fluids, edited by M. BAus et al. (Kluwer Academic Publishers) 1995.

[6] Mao Y., Cates M. E. and Lekkerkerker H. N. W., Phys. Rev. Lett., 75 (1995) 4548; Mao Y., Bladon P., Lekkerkerker H. N. W. and Cates M. E., Mol. Phys., 92 (1997) 151.

[7] Poulin P., Stark H., Lubensky T. C. and Weitz D. A., Science, 275 (1997) 1770.

[8] Zwanzig R., J. Chem. Phys., 39 (1963) 1714.

[9] Evans R., Adv. Phys., 28 (1979) 143.

[10] Chen Z. Y., Phys. Rev. E, 47 (1993) 3765.

[11] Poniewierski A., Phys. Rev. E, 47 (1993) 3396.

[12] Chen Z. Y. and Cui S., Phys. Rev. E, 52 (1995) 3876.

[13] Sheng P., Phys. Rev. A, 26 (1982) 1610; Sluckin T. J. and Poniewierski A., Fluid Interfacial Phenomena, edited by C. A. Croxton (Wiley) 1986.

[14] Frenkel D. and Smit B., Understanding Molecular Simulations: From Algorithms to Applications (Academic Press, Cambridge) 1996.

[15] For $c_{\mathrm{I}}-c_{\mathrm{b}} \geq 10^{-2}$ the variation of $\Gamma$ is very similar to that found in theory. It is not practicable to go much closer to $c_{\mathrm{I}}$ in simulation. 